EPJ Web of Conferences 116, 04001 (2016)

DOI: $10.1051 /$ epjconf/201611604001

(C) Owned by the authors, published by EDP Sciences, 2016

\title{
Search for spatial and temporal collective effects in the ANTARES neutrino telescope data
}

\author{
Alexis Coleiro ${ }^{\mathrm{a}}$, Rodrigo Gracia Ruiz ${ }^{\mathrm{b}}$, Antoine Kouchner ${ }^{\mathrm{c}}$ on behalf of the ANTARES collaboration \\ APC, Université Paris Diderot, CNRS/IN2P3, CEA/Irfu, Observatoire de Paris, Sorbonne Paris Cité, \\ 10 rue Alice Domon et Léonie Duquet, 75205 Paris Cedex 13, France
}

\begin{abstract}
We investigate potential collective effects in the spatial and temporal domains in ANTARES data sets. On the one hand, we apply a two-point correlation analysis to look for inhomogeneities in the arrival directions of the high energy muon neutrino candidates detected between 2007 and 2012. This enables us to provide constraints on models of a population of point sources too faint to be detected by a likelihood-based method. On the other hand, we perform a search for ANTARES neutrino events in temporal coincidence with IceCube High-Energy Starting Events located within $45^{\circ}$ from the Galactic Center. This study, also based on a two-point correlation function, is sensitive to transient emission and does not a prior on either the burst timing structure or on the electromagnetic emission. Therefore, it provides an effective way to acquire information on the possible origin of the IceCube astrophysical signal from transient sources.
\end{abstract}

\section{Introduction}

High energy neutrinos are expected to be produced in sources of cosmic rays. Since they are neutral, weakly interacting and traveling straight from their source without suffering from absorption, neutrinos are unique messengers to further understand the particle acceleration processes in astrophysical sources.

One way of looking for an astrophysical neutrino signal, is to search for clustering in the arrival directions of the reconstructed events. This can be achieved with spatial autocorrelation analysis applied to discrete data ensembles. In the present analysis the method is performed with five years of data and used to search for a signal coming from sources that are too faint to be detected by likelihood analyses.

Furthermore, when dealing with transient emission, the atmospheric background can be significantly reduced using a time-dependent approach. It was pointed out recently that two of the IceCube HighEnergy Starting Events (HESE, see [1]) occurred close to the Galactic Center within 1 day of each other with a p-value of $1.6 \%$ (see [4]). This was interpreted as the possible signature of a transient point source of very-high energy neutrinos in this part of the sky. Consequently, we carry out a model-independent

\footnotetext{
ae-mail: coleiro@apc.in2p3.fr

be-mail: rgracia@apc.in2p3.fr

ce-mail: kouchner@apc.univ-paris7.fr
}

This is an Open Access article distributed under the terms of the Creative Commons Attribution License 4.0, which permits unrestricted use, distribution, and reproduction in any medium, provided the original work is properly cited. 

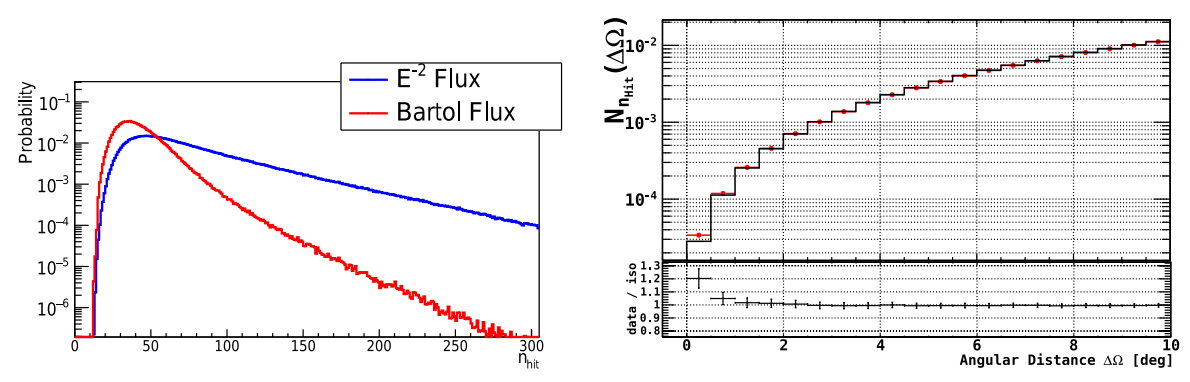

Figure 1. Left: Distributions of the $n_{\text {hit }}$ energy estimator for simulated atmospheric neutrinos, following a Bartol flux $\left(\sim E^{-3.7}\right)$, and simulated cosmic neutrinos following an $E^{-2}$ spectrum. Right: Cumulative autocorrelation distribution for the 2007-2012 ANTARES data set (red points) and for the average randomized ensemble (black line).

analysis based on the timing properties of both the ANTARES and IceCube HESE events to constrain a potential transient neutrino signal in the Galactic Center region.

\section{Search principles}

\subsection{Spatial autocorrelation}

The autocorrelation analysis allows to find inhomogeneities within a discrete data set by studying the two point correlation distribution, which is defined as the distribution of the number of pairs of events as a function of their mutual angular distance $\Delta \Omega$. As it was shown in [2] and [3], weights based on some energy estimator $\bar{E}$ can be applied to the events in order to discriminate between atmospheric neutrinos, which dominate at lower energies, and cosmic neutrinos, of which the spectral distribution in energy is harder (see Fig. 1, left). Formally, the cumulative autocorrelation distribution can be defined as

$$
\mathcal{N}_{n_{H i t}}(\Delta \Omega)=\sum_{i=1}^{N} \sum_{j=i+1}^{N} \omega_{i j} \cdot\left[1-H\left(\Delta \Omega_{i j}-\Delta \Omega\right)\right],
$$

where $H$ is the Heaviside step function and $\omega_{i j}=\omega_{i} \cdot \omega_{j}$ are weights assigned to the couple of

events $i$ and $j$. Each of the individual weights $\omega_{i}$ is defined as $\omega\left(\bar{E}_{i}\right)=\int_{0}^{\bar{E}_{i}} f(\bar{E}) d \bar{E}$, and $f(\bar{E})$ is the normalized distribution of the energy estimator, which can be obtained from Monte Carlo simulations of the atmospheric background. As it is shown in [2], the selected choice for the energy estimator is the number of hits used in the event reconstruction, $n_{\text {hit }}$. Figure 1 left, shows a comparison between the $n_{\text {hit }}$ distribution for atmospheric and astrophysical neutrinos.

A comparison of the autocorrelation function resulting from measured events with the one corresponding to a randomized sample will allow to detect possible clusters of events if a statistically significant excess with respect to isotropy is present in the data.

\subsection{Time correlation}

In the time correlation study, we extend the two-point correlation function to the time domain. Thus, we define the two-point cumulative distribution as

$$
\mathcal{N}(\Delta t)=\sum_{i=1}^{N_{I C}} \sum_{j=1}^{N_{A N T}} \omega_{i j} \cdot\left[1-H\left(\Delta t_{i j}-\Delta t\right)\right],
$$


where $H$ is the Heaviside step function depending on the absolute value of the temporal distance $\Delta t$. $\omega_{i j}$ are the weights assigned to each couple of IceCube (IC) and ANTARES (ANT) events, labelled respectively $i$ and $j$. Each weight $\omega_{i j}$ is computed according to a normal distribution centered on the

IceCube HESE events and defined as $\omega_{i j}=\exp \left(\frac{-\Delta \Omega_{i j}^{2}}{2 \sigma_{i}^{2}}\right)$, where $\Delta \Omega_{i j}$ is the angular distance between each couple of events, and $\sigma_{i}$ is the standard deviation of the $i^{\text {th }}$ IceCube HESE angular error distribution.

\section{Spatial autocorrelation}

\subsection{The ANTARES data set and the reference autocorrelation distribution}

For the present analysis, a data set recorded by the ANTARES neutrino telescope between 2007 and 2012 has been used. The sample contains 5243 up-going neutrino candidates that satisfy selection criteria optimized in order to obtain the best average upper limit on the flux of neutrinos coming from point like sources and extends the data set of the previous analysis (see [2]) by about $50 \%$. The reference autocorrelation distribution has been built as the average of the autocorrelation distributions derived from about $10^{4}$ scrambled data sets. Each of them was obtained by keeping the local coordinates of one neutrino candidate, but assigning it a time randomly selected from another event. This process allows to build an isotropic sky map with the same coverage as the original data set, and in which the non uniformity in the data taking conditions is taken into account.

\subsection{Results and discussion}

The spatial autocorrelation analysis showed that the largest difference between the distributions corresponds to an excess of the ANTARES neutrino candidates with respect to the isotropic ensemble, at scales $<0.5^{\circ}$ (see Fig. 1, right). A statistical comparison following the same method described in [2] was made, and led to the conclusion that this corresponds to a $\sim 2.3 \sigma$ excess. This difference is therefore not significant. Upper limits are currently being derived on the cumulative flux of unresolved sources.

\section{Time correlation with IceCube HESE events}

\subsection{Data samples}

Among the three-year HESE data set, consisting of thirty-seven events (see [1]), nine of them are located within $45^{\circ}$ from the GC, of which eight occur between May 2010 and November 2012. Moreover, the ANTARES data set, between May 2010 and November 2012, has been optimized through Monte-Carlo simulations to reach a constant neutrino candidate rate over time. That was performed by taking into account variations of the mean counting rate of the optical modules due to the evolution of the data taking conditions in the sea. The final ANTARES data set consists of 4337 events.

\subsection{Reference distribution}

To detect correlated structures in the data set, a reference cumulative correlation distribution has been built by averaging $10^{4}$ pseudo-experiments. For each pseudo-experiment, the event times are generated following the time dependent background fluctuations while the event local coordinates are drawn from the local coordinates distribution of the data.

A timing correlation between ANTARES events and IceCube HESE events is searched for by comparing, bin-by-bin, the cumulative two-point distribution of the data with the background distribution. Time correlation features will thus appear as differences between these two distributions. 

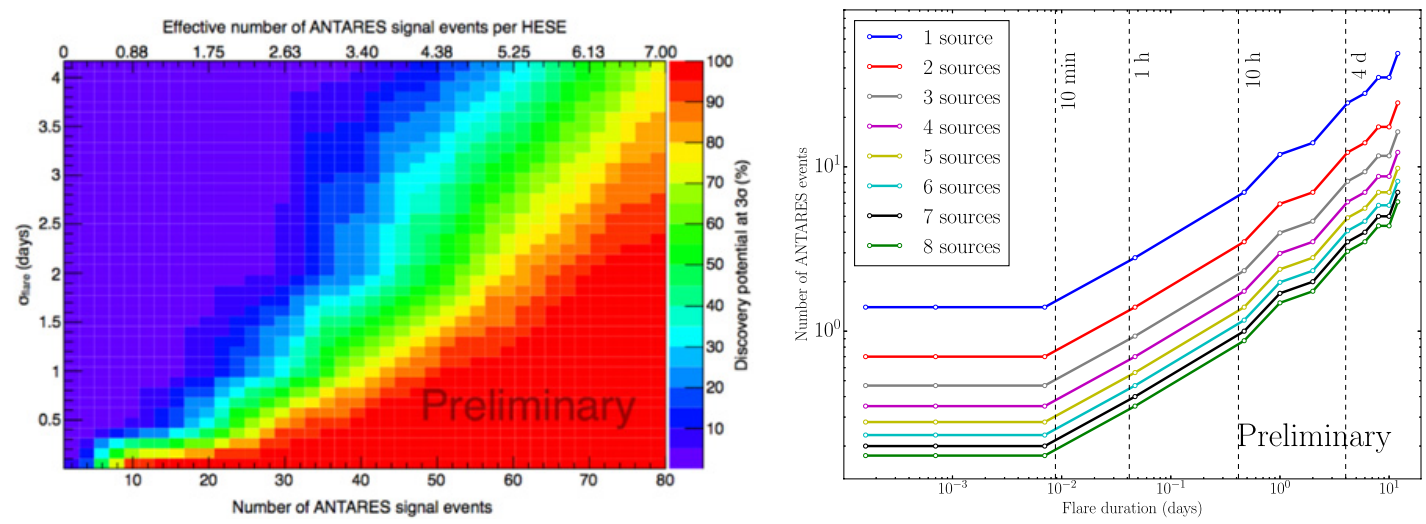

Figure 2. Left: Discovery potential at $3 \sigma$ with respect to the flare duration $\left(\sigma_{\text {flare }}\right)$ and the total number of signal events. The effective number of signal events per IC HESE is also given in the upper part. Right: $90 \%$ confidence level upper limit on the number of ANTARES events temporally correlated with the IceCube HESE events as a function of both the flare duration and the assumed number of sources.

In order to estimate the detection power of the analysis, we performed pseudo-experiments in which one or more event(s) is (are) replaced with one or more signal event(s) that would have come from a transient astrophysical source. The probability for a $3 \sigma$ effect is provided with respect to the different combinations of flare duration and number of generated signal events (see Fig. 2, left plot).

\subsection{Results and discussion}

For the unblinded ANTARES data set, a $\sim 0.9 \sigma$ effect is observed after having performed a statistical comparison similar to the spatial autocorrelation one. Thus, no significant correlation has been found. Consequently, we set upper limits on such a time correlation. Figure 2 (right plot) provides the 90\% confidence level upper limit on the number of ANTARES events temporally correlated with the IceCube HESE events as a function of both the flare duration and the number of sources.

\section{References}

[1] M. G. Aartsen et al., Phys. Rev. Lett., 113, 10, 2014

[2] S. Adrian-Martinez et al., JCAP05, 0001, 2014

[3] ANTARES Collaboration, ICRC 2013 Proc., arXiv: 1312.4308

[4] Y. Bai et al., Phys. Rev. D 90, 6, 2014 